\title{
Age-Dependent, Non-Cell-Autonomous Deposition of Amyloid from Synthesis of $\beta$-Amyloid by Cells Other Than Excitatory Neurons
}

\author{
Karthikeyan Veeraraghavalu, ${ }^{1}$ Can Zhang, ${ }^{2}$ Xiaoqiong Zhang, ${ }^{1}$ Rudolph E. Tanzi, ${ }^{2}$ and Sangram S. Sisodia ${ }^{1}$ \\ ${ }^{1}$ Department of Neurobiology, University of Chicago, Chicago, Illinois 60637, and ${ }^{2}$ Department of Neurology, Genetics and Aging Research Unit, \\ Massachusetts General Institute for Neurodegenerative Disease, Massachusetts General Hospital and Harvard Medical School, Charlestown, Massachusetts \\ 02129-2060
}

Rare, familial, early-onset autosomal dominant forms of familial Alzheimer's disease (FAD) are caused by mutations in genes encoding $\beta$-amyloid (A $\beta$ ) precursor protein (APP), presenilin-1 (PS1), and presenilin-2. Each of these genes is expressed ubiquitously throughout the CNS, but a widely held view is that excitatory neurons are the primary (or sole) source of the A $\beta$ peptides that promote synaptic dysfunction and neurodegeneration. These efforts notwithstanding, APP and the enzymes required for A $\beta$ production are synthesized by many additional cell types, and the degree to which those cells contribute to the production of A $\beta$ that drives deposition in the CNS has not been tested. We generated transgenic mice in which expression of an ubiquitously expressed, FAD-linked mutant PSEN1 gene was selectively inactivated within postnatal forebrain excitatory neurons, with continued synthesis in all other cells in the CNS. When combined with an additional transgene encoding an FAD-linked APP "Swedish" variant that is synthesized broadly within the CNS, cerebral $\mathrm{A} \beta$ deposition during aging was found to be unaffected relative to mice with continued mutant PS1 synthesis in excitatory neurons. Thus, $\mathrm{A} \beta$ accumulation is non-cell autonomous, with the primary age-dependent contribution to cerebral $\mathrm{A} \beta$ deposition arising from mutant PS1-dependent cleavage of APP within cells other than excitatory neurons.

Key words: amyloid deposition; APP; dementia; mouse model; neurodegeneration; presenilin

\section{Introduction}

Alzheimer's disease (AD) is a progressive neurodegenerative disease characterized by impairments in cognition and memory, neuronal death, and deposition of 40-42 amino acid $\beta$-amyloid $(\mathrm{A} \beta)$ peptides that are derived from larger amyloid precursor proteins (APPs). Familial, early-onset, autosomal dominant forms of $\mathrm{AD}(\mathrm{FAD})$ are caused by inheritance of mutant genes encoding APP, presenilin-1 (PS1), and presenilin-2 variant polypeptides that are expressed ubiquitously in all CNS cell types and peripheral organs. Presenilins are the catalytic subunits of the $\gamma$-secretase complex that promotes intramembranous proteolysis of APP and a number of type I membrane proteins (De Strooper, 2003). Importantly, FAD-linked mutant forms of pre-

Received Dec. 4, 2013; revised Jan. 13, 2014; accepted Feb. 4, 2014.

Author contributions: K.V., C.Z., R.E.T., and S.S.S. designed research; K.V., C.Z., X.Z., and R.E.T. performed research; K.V., C.Z., R.E.T., and S.S.S. contributed unpublished reagents/analytic tools; K.V., C.Z., R.E.T., and S.S.S. analyzed data; K.V., C.Z., R.E.T., and S.S.S. wrote the paper.

This work was supported by National Institutes of Health Grants AG021494 and AG027854 (S.S.S.), the Cure Alzheimer's Fund (S.S.S.), the Edward H. Levi Fund (K.V.), and the Adler Foundation (K.V., S.S.S.). We thank Dr. Vytas Bindokas, Microscopy Core Facility, for expert support with image analysis.

S.S.S. is a paid consultant of Eisai Research Labs, AZ Therapies, and Jannsen Pharmaceutica but is not a shareholder in any company that is a maker or owner of a Food and Drug Administration-regulated drug or device. The other authors declare no competing financial interests.

Correspondence should be addressed to Dr. Sangram S. Sisodia, University of Chicago, Department of Neurobiology, 947 East 58th Street, AB 308, Chicago, IL 60637. E-mail: ssisodia@bsd.uchicago.edu.

DOI:10.1523/JNEUROSCI.5079-13.2014

Copyright $\odot 2014$ the authors $\quad 0270-6474 / 14 / 343668-06 \$ 15.00 / 0$ senilins cause disease by elevating the ratio of $\mathrm{A} \beta_{42} / \mathrm{A} \beta_{40}$ peptides, ultimately leading to early and selective cerebral deposition of $\mathrm{A} \beta_{42}$ peptides (Price and Sisodia, 1998).

Neuronal-specific overexpression of FAD-linked APP or PSEN1 transgenes leads to cerebral $\mathrm{A} \beta$ deposition in transgenic mice but attempts to evaluate the effect on $A \beta$ metabolism or deposition of expressing these mutant genes in other CNS cell types has not been forthcoming. To explore the effect on $\mathrm{A} \beta$ levels and deposition, we now report the outcome of selective excision of a widely expressed mutant PSEN1 $\triangle E 9$ transgene within excitatory neurons, because these cells are known to produce high levels of $\mathrm{A} \beta$ peptides in an activity-dependent manner (Kamenetz et al., 2003). Despite $>80 \%$ reduction in steady-state $\mathrm{PS} 1 \Delta \mathrm{E} 9$ polypeptide levels in the brain by the age of 7 months, age-dependent accumulation of $\mathrm{A} \beta$ and amyloid deposition (at 12 months of age) was unaffected, and the levels of soluble and insoluble $\mathrm{A} \beta$ peptides in younger mice ( $8-9$ months) were only modestly affected. However, loss of mutant presenilin expression by excitatory neurons did markedly suppress $\mathrm{A} \beta$ deposition in early adult life during aging (between 5 and 7 months of age). We further demonstrate that cultured astrocytes and microglia secrete high levels of $\mathrm{A} \beta$ peptides. Thus, during aging of this mouse model, cells other than excitatory neurons provide a sustained source of secreted $\mathrm{A} \beta$ peptides that may accrete onto "seeds" that form independent of $A \beta$ production by excitatory neurons and/or serve as templates for seeding and aggregation at later stages of amyloidogenesis. 
A

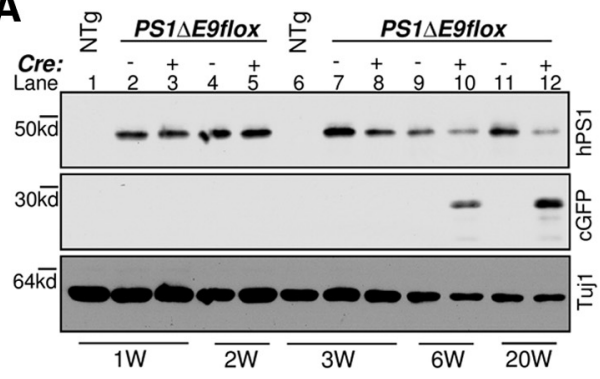

D

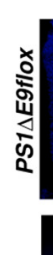

B
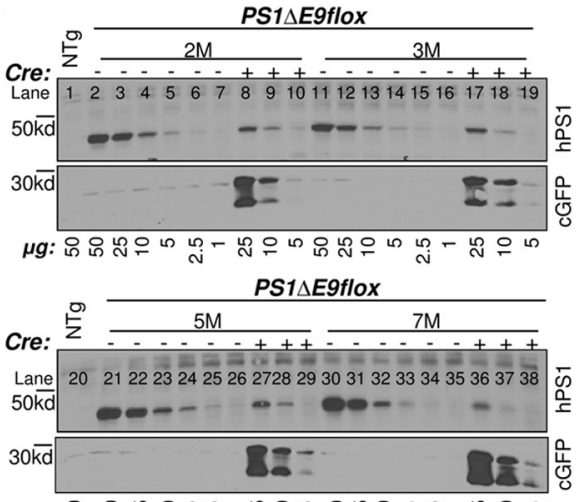

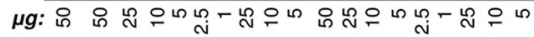

C

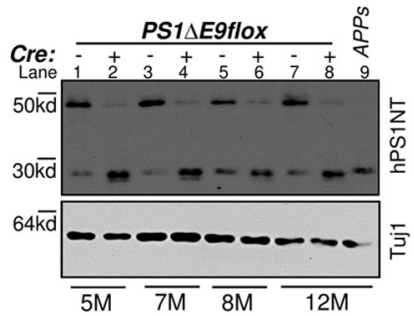

E
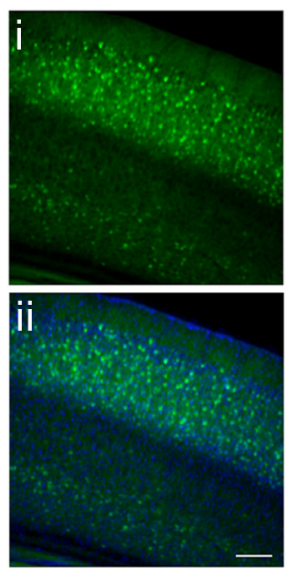

$5 \mathrm{M}$

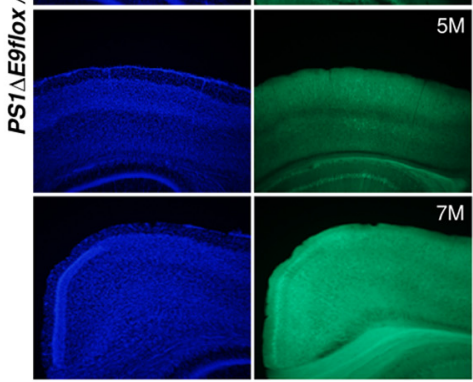

DAPI

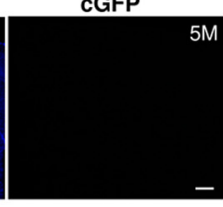

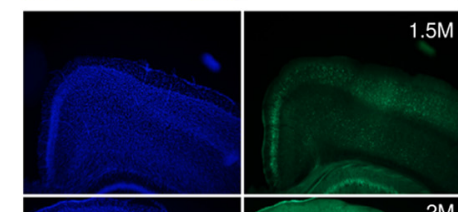

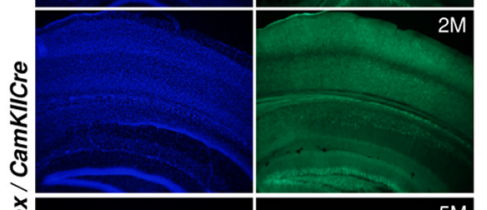

G

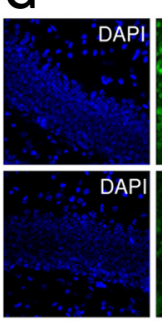

$\mathbf{F}$

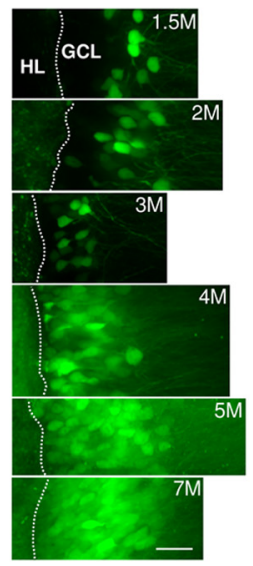

overlay
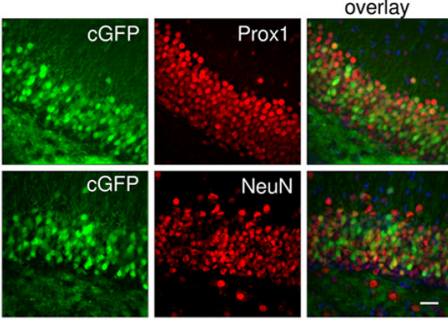

Figure 1. Postnatal deletion of PS1 $\Delta$ E9 transgene expression in PS1 $\Delta$ E9flox/CaMKIICre mice. A, Total brain protein lysates from 1-, 2-, 3-, 6-, or 20-week-old mice were immunoblotted (20 $\mu \mathrm{g} /$ lane) with antibodies specific to human PS1 (hPS1), CGFP, or $\beta$ III-tubulin (Tuj1). One- or 3-week-old NTg brain lysate was loaded as controls. $\boldsymbol{B}$, Total protein lysates from NTg mice (50 $\mu$ g), PS1 $\Delta$ E9flox mice $(50,25,10,5,2.5,1 \mu \mathrm{g})$, or PS1 $\Delta$ E9flox/CaMKIICre mice $(25,10,5 \mu \mathrm{g})$ at 2, 3, 5, or 7 months immunoblotted with antibodies specific to human PS1 (hPS1) or cGFP. Equal protein content of $50 \mu \mathrm{g} /$ lane was loaded by supplementing the samples from different dilutions with NTg protein lysate. C, Total brain protein lysates prepared from 5 , 7, 8, or 12 month age-matched PS1 $\Delta$ E9flox mice or PS1DE9flox/CaMKIICre mice were immunoblotted (20 $\mu \mathrm{g} /$ lane) with PS1-NT antibody (hPS1NT). Brain lysate from 12-month-old APPswe mice was loaded as control. D, Images on the right reveal CGFP expression (green), turned on by Cre-mediated excision of PS1 $\Delta$ E9 cassette. Gradual increase in endogenous green fluorescence signal was observed within the cortex of PS1 $\triangle$ E9flox/CaMKIICre mice brain sections at 1.5, 2, 5, and 7 months of age. No cGFP expression was detected in cortex or hippocampus of 5-month-old PS1 $\triangle$ E9flox mice brain section (top row). Images on the left are the respective DAPI-stained images. Scale bar, $250 \mu \mathrm{m}$. E, Confocal image reveals that neurons located in the layers III, V, and VI expressed cGFP in PS1 $\triangle$ E9flox/CaMKIICre mice by 3 months of age (Ei), and the overlay with DAPI counterstain is shown below (Eii). Scale bar, $100 \mu \mathrm{m}$. $\boldsymbol{F}$, Confocal image of dentate gyrus in PS1 $\Delta$ E9flox/CaMKIICre mice brain sections at 1.5, 2 , 3,5 , and 7 months of age. Progressive expression of cGFP was observed in the cell bodies and neurites of granule cell neurons in the granule cell layer (GCL) from the outermost layers to innermost layers of the dentate gyrus. HL, Hilus. Scale bar, $10 \mu \mathrm{m}$. G, Images of dentate gyrus from 4-month-old PS1DE9flox/CaMKIICre mice, double stained with antibodies specific to neuronal markers Prox1 (red) or NeuN (red). cGFP-expressing dentate granule cells were colabeled with Prox1 and NeuN. Scale bar, $25 \mu \mathrm{m}$. M, Month; W, week.

\section{Materials and Methods}

Animals. Male mice expressing prion promoter (PrP)-driven PS1 transgenes [PrP.APPswe (Borchelt et al., 1997), PrP.PS1DE9flox (line 13; Veeraraghavalu and Sisodia, 2013), and $\alpha$ CaMKIICre (line T29-2; Tsien et al., 1996)] were maintained heterozygous for the transgene in $(\mathrm{C} 3 \mathrm{H} /$ $\mathrm{HeJ} \times \mathrm{C} 57 \mathrm{BL} / 6 \mathrm{~J} \mathrm{~F} 3) \times \mathrm{C} 57 \mathrm{BL} / 6 \mathrm{~J}$ n1 background. Mice homozygous for PS1 1 E9flox and $\alpha$ CaMKIICre transgenes were crossed to PrP.APPswe mice to generate APPswe/PS1 $\triangle E 9$ flox/CaMKIICre mice carrying all three transgenes. Animal experiments were conducted in accordance with institutional approval and National Institutes of Health guidelines.

Tissue preparation, Western blot analyses, immunocytochemistry, and sandwich ELISA assays. Animals were deeply anesthetized and perfused transcardially with cold $0.9 \%$ saline solution, and brains were harvested. One hemisphere was snap frozen for biochemical analysis, and the other half was fixed in $4 \%$ paraformaldehyde and transferred into $30 \%$ sucrose; $40-\mu \mathrm{m}$-thick coronal sections were stored at $-20^{\circ} \mathrm{C}$ in cryoprotective buffer before histology. For Western blot analysis, brain tissue was homogenized in buffer containing $1 \%$ SDS and protein concentration in the soluble fraction determined using BCA protein assay (Pierce). For immunofluorescence studies, every 6th or 12th coronal section spanning the proximal to distal end of the hippocampus including cortical regions was used. Sections were incubated with $\mathrm{A} \beta$-specific 3D6 antibody and bound antibody visualized using a fluorescently labeled secondary antibody. Amyloid area fraction and plaque numbers were quantified using NIH Image J, as described previously (Veeraraghavalu et al., 2013). Data are reported as mean \pm SEM. Student's $t$ test (unpaired) was performed for comparisons of quantitative data. Values of $p<0.05$ were used as the criterion for statistical significance. The levels of TBS-soluble and 70\% formic acid-soluble $A \beta_{40}$ and $A \beta_{42}$ peptides were determined using ELISA protocols as described previously (Veeraraghavalu et al., 2013).

\section{Results}

Postnatal deletion of mutant PSEN1 in forebrain excitatory neurons

In previous studies, we characterized a transgenic mouse line harboring a PrP.PS1 $\Delta E 9^{\text {lox/lox }}$.cGFP transgene (PS1DE9flox 
A
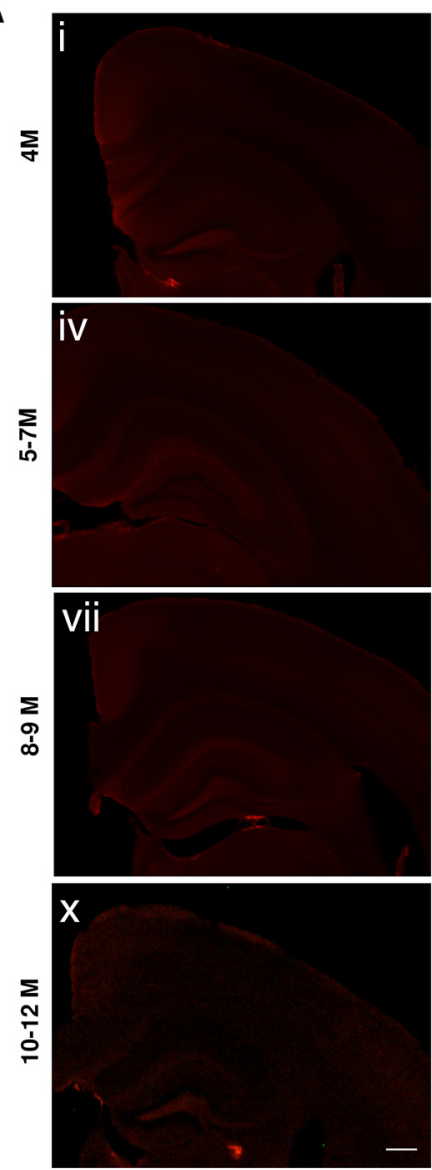

B
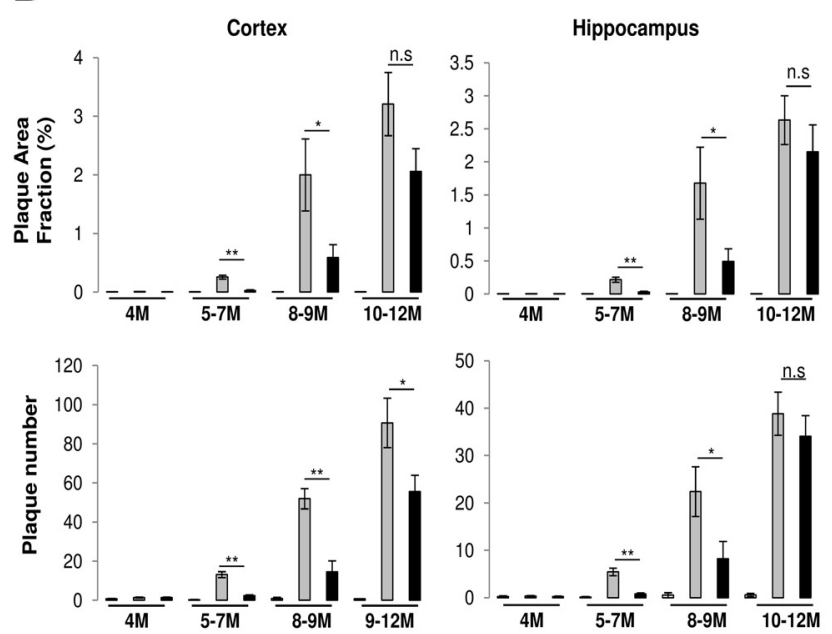
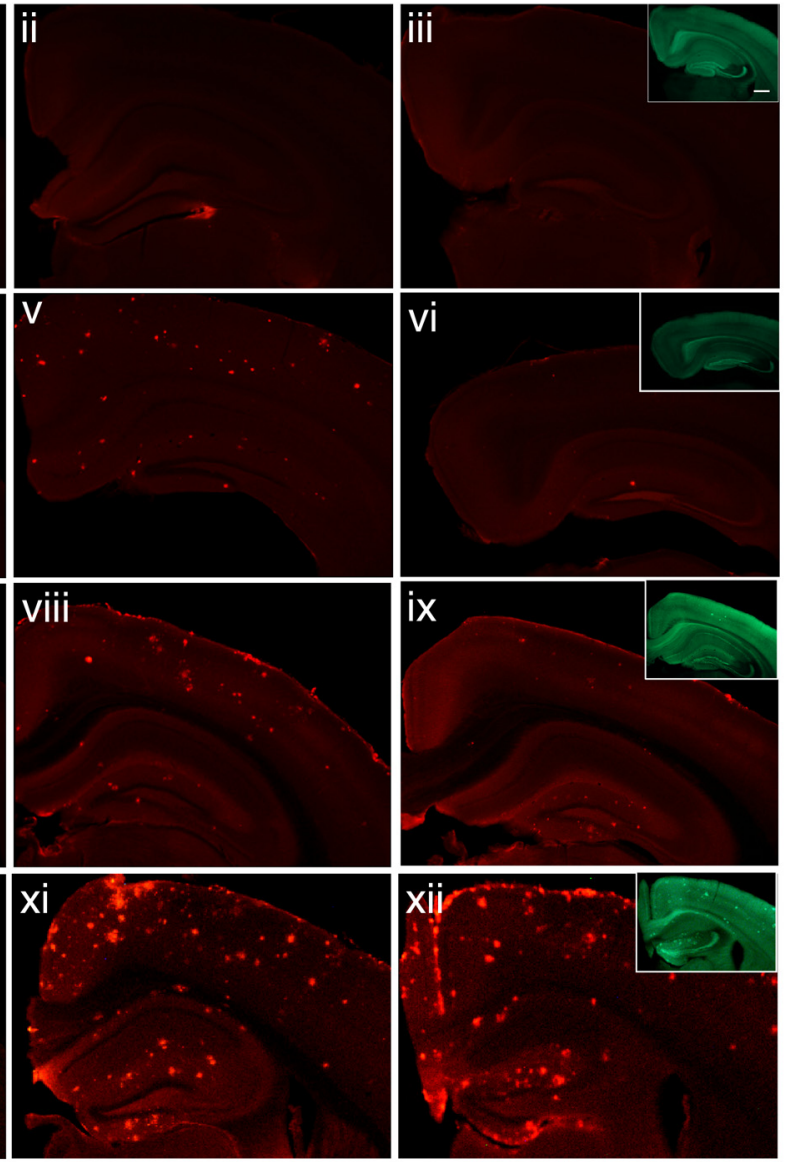

C
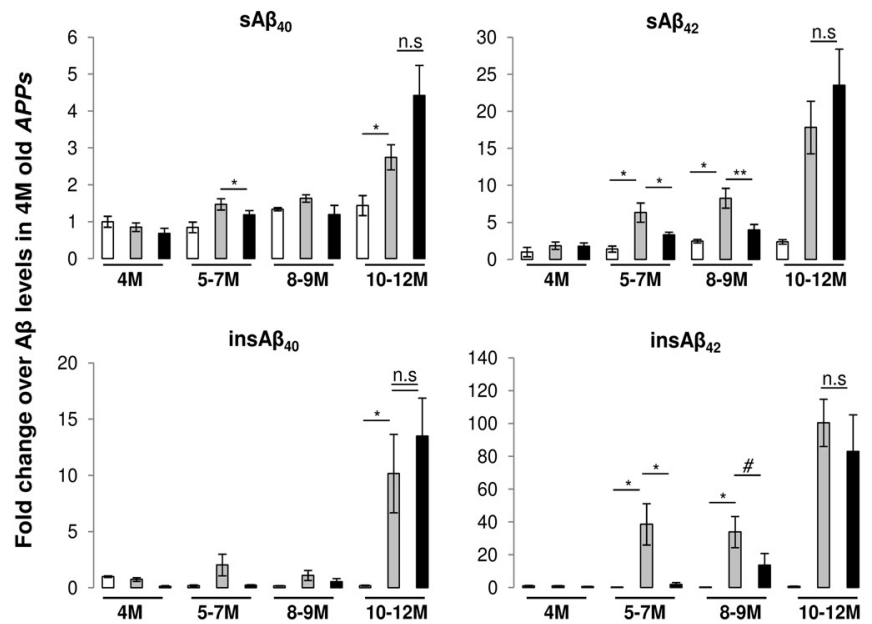

Figure 2. Amyloid deposition and A $\beta$ levels as a function of age in brains of $A P P$ swe, APPswe/PS1 $\triangle$ E9flox, or APPswe/PS1 $\triangle E 9 f l o x / C a M K I I C r e$ mice. $A$, Extent of amyloid deposition after postnatal ablation of FAD-linked mutant PS1 expression in mature excitatory neurons. Images of hemibrain sections from APPswe (Ai, Aiv, Avii, Ax), APPswe/PS1 $\triangle$ E9flox (Aii, Av, Aviii, Axi), or APPswe/ PS1DE9flox/CaMKIICre (Aiii, Avi, Aix, Axii) mice aged to 4, 5-7, 8-9, or 10-12 months immunostained with $3 D 6$ (red). cGFP expression as observed by cGFP fluorescence (green) in APPswe/ PS1 $\triangle$ E9flox/CaMKIICre mice are shown within the insets in Aiii, Avi, Aix, and Axii. Scale bar, $500 \mu \mathrm{m}$. B, Quantification of amyloid deposition after postnatal ablation of FAD-linked mutant PS1 expression in mature excitatory neurons. Histograms show the amyloid plaque area fraction (percentage) and plaque numbers in the cortex or hippocampus of $A P P$ swe, APPswe/PS1 $\triangle$ E9flox, or APPswe/PS1 $\triangle$ E9flox/CaMKIICre mice aged to 4, 5-7, 8-9, or 10-12 months. $n=6$ (4 months, APPswe), 7 (4 months, APPswe/PS1 months, APPswe), 8 (5-7 months, APPswe/PS1 $\triangle$ E9flox; plaque area fraction in the cortex and hippocampus was $0.255 \pm 0.033$ and $0.218 \pm 0.039 \%$, respectively, and the plaque number was $13.15 \pm 1.49$ and $5.47 \pm 0.79$, respectively), 8 (5-7 months, APPswe/PS1 $\Delta$ E9flox/CaMKIICre), 3 (8-9 months, APPswe), 6 (8-9 months, APPswe/PS1 $\Delta$ E9flox; plaque area fraction was $1.99 \pm$ 0.61 and $1.67 \pm 0.54 \%$, respectively, and plaque numbers were $51.96 \pm 5.18$ and $22.4 \pm 5.24$, respectively), 7 ( $8-9$ months, APPswe/PS1 $\Delta$ E9flox/CaMKI/Cre; plaque area fraction in the cortex and hippocampus was $0.589 \pm 0.21$ and $0.49 \pm 0.19 \%$, respectively, and plaque numbers were $14.57 \pm 5.64$ and $8.22 \pm 3.64$, respectively), 3 ( $10-12$ months, APPswe), 4 ( $10-12$ months, APPswe/PS1 $\triangle$ E9flox; plaque area fraction and plaque numbers in cortex were $3.2 \pm 0.53 \%$ and $90.7 \pm 12.6$, respectively, and the plaque area fraction and plaque numbers in hippocampus were $2.62 \pm 0.36 \%$ and $38.85 \pm 4.55$, respectively), and 5 ( $10-12$ months, APPswe/PS1 $\triangle$ E9flox/CaMKIICre; plaque area fraction and plaque number in the cortex were $2.05 \pm 0.39 \%$ and $55.6 \pm 8.36$, respectively, and the plaque area fraction and plaque numbers in the hippocampus were $2.15 \pm 0.41 \%$ and $34.08 \pm 4.35$, respectively). Plaque area fraction in the cortex: ${ }^{*} p=0.021$ for $8-9$ months and ${ }^{* *} p=0.00013$ for $5-7$ months for APPswe/PS1 $\Delta$ E9flox versus APPswe/PS1 $\Delta$ E9flox/CaMKIICre groups.Plaque numbers in the cortex: ${ }^{*} p=0.023$ for $10-12$ (Figure legand continues.) 
mice), wherein the ubiquitously expressed murine PrP drives expression of a mutant PS1 $\triangle E 9$ cDNA flanked by loxP sites and includes a downstream cDNA encoding the copepod green fluorescent protein (cGFP; Veeraraghavalu and Sisodia, 2013); in $P S 1 \Delta E 9 f l o x$ mice, expression of the PS1 $\triangle E 9$ transgene occurs in all neuronal and non-neuronal cell types, leaving expression of the cGFP reporter silent. Cell-type-specific expression of Cre recombinase results in excision of the PS1 $\triangle E 9$ cDNA cassette, thus activating expression of the cGFP reporter in cells wherein recombination has occurred (Veeraraghavalu and Sisodia, 2013).

To examine the roles of excitatory neurons on amyloid deposition and $\mathrm{A} \beta$ production, we crossed the PS1 $\Delta$ E9flox mice with $\alpha$ CaMKIICre transgenic mice (line T29-1; (Tsien et al., 1996), wherein Cre recombinase is expressed postnatally and is restricted to excitatory neurons of the forebrain. Western blot analysis of detergent solubilized brain lysates prepared from either 1-, 2-, 3-, 6-, or 20-week-old PS1 2 E9flox mice or PS1 1 E9flox/ CaMKIICre mice were performed with a rat monoclonal antibody specific for human PS1 (Lah et al., 1997) or cGFP (Fig. 1A). Compared with 1- or 3-week-old nontransgenic (NTg) mice, expression of the PS1 $\mathrm{E} 9$ polypeptide was observed in PS1 1 E9flox mice at all ages (Fig. 1A, compare lanes 1, 6 with 2, 4, 7, 9, 11). However, compared with PS1 $\Delta$ E9flox mice (Fig. 1A, lanes 2-5), a prominent reduction in PS1 $\Delta \mathrm{E} 9$ polypeptide levels was evident in PS1 $\mathrm{E}$ 9flox/CaMKIICre mice beginning at 6 weeks of age (Fig. $1 A$, compare lanes 9 with 10 and 11 with 12). In line with these observations, cGFP reporter expression was observed only in lysates prepared from PS1 $\mathrm{E9}$ flox/CaMKIICre mice beginning at 6 weeks of age (Fig. 1A, lanes 10, 12, cGFP panel).

To semiquantitatively determine the extent of reduction in PS1 1 E9 levels, human PS1-specific antibody was used in Western blot analysis of serial diluted total protein lysates prepared from the hippocampi of age-matched PS1 E E9flox or PS1 E Eflox/ CaMKIICre mice (Fig. $1 B$ ). These studies reveal that, in 2-, 3-, 5-, or 7-month-old PS1 $\Delta$ E9flox/CaMKIICre mice, PS1 $\Delta \mathrm{E} 9$ polypeptide levels are reduced by $48,58.3,69.7$, and $78.5 \%$, respectively, relative to the levels seen in PS1 $\triangle E 9$ flox mice. We speculate that the residual PS1 $\Delta \mathrm{E} 9$ signal in the PS1 $\mathrm{E} 9$ flox/CaMKIICre mice samples represents mutant PS1 expression in cells that do not express Cre. Western blot analysis of protein lysates prepared from the brains of 5-, 7-, 8-, or 12-month-old PS1 1 E9flox using polyclonal PS1-amino terminal (NT) antibody that recognizes both human and mouse PS1 (Thinakaran et al., 1997) revealed that the endogenous mouse N-terminal derivative (NTF) is "replaced" (Fig. 1C, lanes 1, 3, 5, 7). Conversely, in brains of 5-, 7-,

$\leftarrow$

(Figure legand continued.) months, ${ }^{* *} p<0.0001$ for $5-7$ months, and ${ }^{* *} p=0.008$ for $8-9$ months for APPSwe/PS1DEgflox versus APPswe/PS1 EEfflox/CaMKIICre groups. Plaque area fraction in the hippocampus: ${ }^{*} p=0.025$ for $8-9$ months and ${ }^{* *} p=0.001$ for $5-7$ months for APPswe/PS1 1 E9flox versus APPswe/PS1DE9flox/CaMKIICre groups. Plaque numbers in the hippocampus: ${ }^{*} p=0.022$ for $8-9$ months and ${ }^{* *} p=0.0004$ for $5-7$ months for APPswe/ PS1 $1 \Delta$ E9flox versus APPSwe/PS1DE9flox/CaMKIICre groups. n.s., Not significant. C, Changes in steady-state levels of $A \beta_{40}$ and $A \beta_{42}$ peptides after postnatal ablation of FAD-linked mutant PS1 expression in mature excitatory neurons. Histograms show fold changes in $\mathrm{SA} \beta_{40}, \mathrm{SA} \beta_{42}$, ins $A \beta_{40}$, or ins $A \beta_{42}$ levels in hemibrains of APPswe, APPswe/PS1 1 E9flox, or APPswe/ PS1DE9flox/CaMKII/Cre mice aged to 4,5-7, 8-9, or 10-12 months over the levels observed in age-matched APPswe mice group. $n=6$ (4 months, APPswe), 7 (4 months, APPswe/ PS1 EEgflox), 7 (4 months, APPswe/PS1 1 E9flox/CaMKII(re), 5 (5-7 months, APPswe), 8 (5-7 months, APPswe/PS1DEgflox), 8 (5-7 months, APPswe/PS1 1 E9flox/CaMKIICre), 2 (8-9 months, APPswe), 5 (8-9 months, APPSwe/PS1 EEgflox), 6 (8-9 months, APPswe/ PS1 1 Egflox/CaMKIICre), 3 (10-12 months, APPswe), 5 (10-12 months, APPswe/PS1 E E9flox), and 5 (10-12 months, APPswe/PS1 EEfflox/CaMKIICre). ${ }^{*} p<0.05,{ }^{* *} p<0.01,{ }^{\#} p=0.058$. n.s., Not significant.
8-, or 9-month-old PS1 $\triangle E 9$ flox/CaMKIICre mice, excision of the PS1 $\Delta$ E9flox cassette led to the accumulation of mouse NTF (Fig. $1 C$, lanes $2,4,6,8$, respectively), as was expected if endogenous PS1 can compete with the limiting components of the $\gamma$-secretase complex (Thinakaran et al., 1997).

Consistent with the Western blot analyses, cGFP fluorescence was observed in the cortex and hippocampus of PS1DE9flox/ CaMKIICre mice beginning at the age of 1.5 months that increased in intensity in an age-dependent manner (Fig. $1 D-F$ ); the cGFP-positive cells express the neuronal antigens Prox1 and/or NeuN but not antigens specific for astrocytes (GFAP and S100 $\beta$ ) or microglia (Iba1) (Veeraraghavalu and Sisodia, 2013).

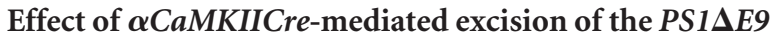 cassette in excitatory neurons on $\mathrm{A} \beta$ deposition and steady- state $\mathrm{A} \boldsymbol{\beta}$ levels}

We first bred PS1 1 E9flox mice to PrP promoter-driven APPswe transgenic mice that express APPswe at $\sim 2.5$-fold over endogenous APP and only exhibit sparse amyloid deposits beginning at $\sim 18-20$ months of age (Borchelt et al., 1997). The resulting APPswe/PS1DE9flox mice were then crossed with CaMKIICre mice to generate APPswe/PS1 $\triangle E 9$ flox/CaMKIICre mice. Cohorts of male APPswe, APPswe/PS1 1 E9flox, or APPswe/PS1DE9flox/ CaMKIICre mice were aged to 4, 5-7, 8-9, or 10-12 months, and the extent of $\mathrm{A} \beta$ deposition was revealed by immunostaining with an $\mathrm{A} \beta$-specific 3D6 monoclonal antibody. As expected, we did not observe 3D6-positive $\mathrm{A} \beta$ deposits in the brains of $A P P$ swe mice aged to 4, 5-7, 8-9, or 10-12 months (Fig. 2Ai,Aiv,Avii,Ax, respectively; quantified in $B$ ) or in the brains of 4 -month-old APPswe/PS1DE9flox and APPswe/PS1DE9flox/CaMKIICre mice (Fig. 2Aii,Aiii; quantified in $B$ ).

Sandwich ELISA analysis of TBS soluble (s) and formic acidextractable insoluble (ins) $\mathrm{A} \beta$ peptides from hemibrains of the same cohorts of animals that were assessed by 3D6 immunocytochemistry (Fig. $2 B$ ) revealed no significant differences in the levels of $\mathrm{sA} \beta_{40}, \mathrm{sA} \beta_{42}$, insA $\beta_{40}$, or insA $\beta_{42}$ between APPswe, APPswe/PS1 1 E9flox, or APPswe/PS1 $D$ E9flox/CaMKIICre mice at 4 months of age (Fig. 2C).

Compared with the 5- to 7-month-old APPswe mice (Fig. $2 A i v)$, the APPswe/PS1 $\triangle E 9$ flox mice exhibited robust amyloid deposition (Fig. $2 A v$; quantified in $B$ ). Supporting these findings, we observed a significant increase in $\mathrm{sA} \beta$ and ins $\beta$ species in APPswe/PS1DE9flox mice compared with APPswe mice (Fig. $2 C)$. Interestingly, in 5- to 7-month-old APPswe/PS1DE9flox/ CaMKIICre mice, we observed a dramatic reduction in 3D6 immunoreactivity compared with the APPswe/PS1DE9flox mice (Fig. 2, compare Avi with $A v$ ); the cortex and hippocampus of APPswe/PS1 1 E9flox/CaMKIICre mice exhibited significant 10.14- and 7.08-fold reductions in plaque area, respectively, and 5.59- and 6.63-fold reduction in plaque number, respectively (Fig. $2 B$ ). Indeed, biochemical analyses revealed a significant decrease in $s A \beta_{42}$ and insA $\beta_{42}$ levels in 5- to 7-month-old APPswel PS1 $\triangle E$ 9flox/CaMKIICre cohorts compared with the age-matched APPswe/PS1 1 E9flox cohorts (Fig. 2C).

At 8-9 months of age, the APPswe/PS1 $\triangle E 9$ flox mice exhibited even higher levels of $A \beta$ deposits compared with the 5- to 7-month-old APPswe/PS1 1 E9flox cohorts, as expected (Fig. 2, compare $A v$ iii with $A v$; quantified in $B$ ). Moreover, we observed a modest reduction in $\mathrm{A} \beta$ deposition in the cortex and hippocampus of the 8- to 9-month-old APPswe/PS1 1 E9flox/CaMKIICre mice compared with their APPswe/PS1 $\triangle E 9$ flox cohorts (Fig. 2, compare Aix with Aviii). Compared with the 8- to 9-month-old APPswe/PS1DE9flox mice, the cortex and hippocampus of 
APPswe/PS1 EE9flox/CaMKIICre mice exhibited a significant 3.38- and 3.39-fold reduction in plaque area, respectively, and a 3.56- and 2.72-fold reduction in plaque number, respectively (Fig. $2 B$ ). These morphological findings were confirmed by A $\beta$ ELISAs (Fig. 2C). Thus, although there appears to be significant reductions in plaque area and number in the brains of 8- to 9-month-old APPswe/PS1 1 E9flox/ CaMKIICre mice compared with their APPswe/PS1DE9flox cohorts of the same age, the extent of reduction is not as dramatic as that observed compared with their respective 5- to 7-month-old cohorts.

At 10-12 months of age, the APPswel PS1 1 E9flox mice exhibited even higher levels of $\mathrm{A} \beta$ deposits compared with the 5 to 7-month-old or 8- to 9-month-old APPswe/PS1 EE9flox cohorts (Fig. 2, compare Axi with Av, Aviii; quantified in $B)$. However, compared with amyloid deposition in 10- to 12-month-old APPswe/PS1 1 E9flox mice (Fig. 2Axi), 10- to 12-month-old APPswe/PS1 $\triangle E 9$ flox/CaMKIICre mice (Fig. $2 A x i i)$ exhibited a very modest 1.56 - and 1.63-fold reduction in plaque area and plaque number in the cortex, respectively, and a 1.22- and 1.13-fold reduction in plaque area and plaque number in the hippocampus, respectively (Fig. 2, compare Axii with Axi; quantified in $B$ ). Indeed, ELISAs failed to detect a statistically significant difference in the levels of sA $\beta$ or insA $\beta$ peptide species in APPswe/PS1 $\triangle E 9$ flox/CaMKIICre mice compared with APPswe/PS1 1 E9flox mice (Fig. 2C).

In summary, although postnatal deletion of the mutant $P S 1 \triangle E 9$ transgene in excitatory neurons results in a significant reduction in both $\mathrm{A} \beta$ deposition and steady-state $\mathrm{A} \beta$ levels in young animals, we failed to observe a reduction in either of these parameters in aged animals.

\section{Generation of $A \boldsymbol{\beta}$ peptides in non-neuronal cells from transgenic mice}

That the PrP promoter-driven transgene is ubiquitously expressed led us to investigate the possibility that the enzymatic machinery responsible for $\mathrm{A} \beta$ generation, namely $\beta$-secretase (BACE1) and presenilin/ $\gamma$-secretase, are present in cells other than excitatory neurons that could contribute to the sustained elevation in steady-state levels of $\mathrm{A} \beta$ observed in older APPswe/ PS1DE9flox/CaMKIICre mice. To address this issue, primary astrocytes, microglia, or adult neural progenitor cultures (NPCs) that are derived from radial glial-like precursors were established from the brains of newborn PS1 $\triangle E 9$ flox or APPswe mice or 8-week-old APPswe/PS1 $\triangle E$ 9flox mice. Conditioned medium (CM) collected from astrocyte (ACM), microglia (MCM), or NPC (NCM) cultures were fractionated by SDS-PAGE and subjected to Western blot analysis using the $\mathrm{A} \beta$-specific monoclonal antibody $26 \mathrm{D} 6$. Secreted $\mathrm{A} \beta$ peptides were readily detected in the $\mathrm{CM}$ of all three cell types established from APPswe or APPswe/ PS1DE9flox mice (Fig. 3A). Treatment of the cultures with a $\gamma$-secretase inhibitor (L-685,458 [1S-benzyl-4R-(1S-carbamoyl-2phenylethylcarbamoyl-1S-3-methylbutylcarbamoyl)-2R-hydroxy-5-phenylpentyl] carbamic acid tert-butyl ester]) abolished $\mathrm{A} \beta$ secretion and resulted in the intracellular accumulation of APP C-terminal fragments, the penultimate substrate for $\mathrm{A} \beta$ generation (Fig. $3 B$ ). Moreover, both $\mathrm{A} \beta_{40}$ and $\mathrm{A} \beta_{42}$ peptides are
B
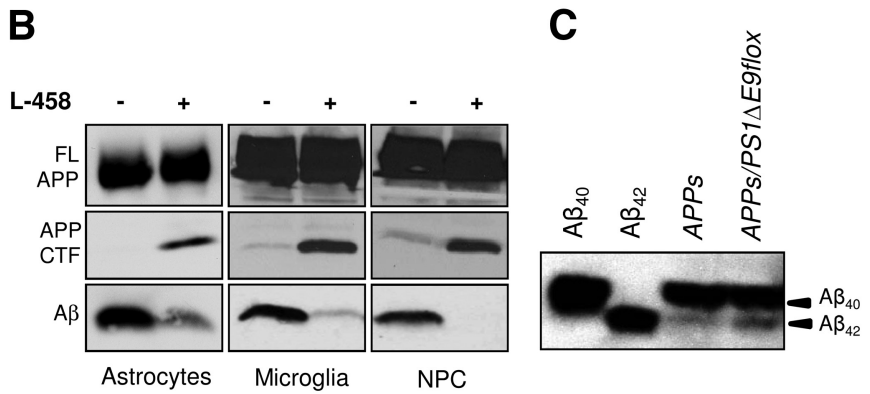
with a notable elevation in $\mathrm{A} \beta_{42}$ peptide levels in APPswe/ PS1 EE9flox MCM (Fig. 3C). Collectively, these results reveal that the enzymes necessary for generating $A \beta$ are present in multiple non-neuronal cell types, findings that would argue that these species contribute to amyloid deposition and steady-state levels of $\mathrm{A} \beta$ peptides in the adult $\mathrm{CNS}$ of our mouse model.

\section{Discussion}

Although a series of preceding efforts established that neuronal overexpression of FAD-linked mutant APP and PSEN1 transgenes promotes CNS deposition of $\mathrm{A} \beta$, it is clear that transcripts and encoded APP and PS1 are expressed in a wide variety of cell types in the adult CNS. For example, PSEN1 mRNA is highly expressed in neurons, astrocytes, and oligodendrocytes in white matter (Sherrington et al., 1995). Moreover, light and immuno-EM approaches revealed that PS1 is expressed in neurons, glial cells, and blood vessels in primate brain (Lah et al., 1997). Similarly, APP isoforms are expressed in non-neuronal cells of human brain white matter (Golde et al., 1990) and in cultured murine and human astrocytes, microglia, and endothelial cells (Haass et al., 1991; Forloni et al., 1992; Mönning et al., 1995). Thus, we sought to examine the effect of expressing FAD-linked mutant genes in CNS cell types other than excitatory neurons.

By selective inactivation of a widely expressed FAD-linked mutant presenilin gene in postnatal forebrain excitatory neurons (a principal source of secreted $\mathrm{A} \beta$ peptides that are released in an activity-dependent manner; Kamenetz et al., 2003; Cirrito et al., $2005)$, we have now demonstrated that the levels of insA $\beta$ peptides and amyloid burden in mice with or without excitatory neuron production of $A \beta$ are indistinguishable by $10-12$ months of age. Thus, production and accumulation of $A \beta$ peptides during aging is independent of $\mathrm{A} \beta$ production by excitatory neurons, an observation that argues persuasively for alternative cellular source(s) and/or factor(s) that contribute to $\mathrm{A} \beta$ deposition in an age-dependent manner. Consistent with a non-neuron contribution, we demonstrate that cultured astrocytes and microglia from APPswe/PS1DE9flox mice secrete robust levels of $\mathrm{A} \beta$ peptides. Moreover, age-dependent, excitatory neuron-independent accumulation of $\mathrm{A} \beta$ occurs despite our evidence that excitatory neurons are a principal source of $\mathrm{A} \beta$ peptides deposited in younger animals. 
Thus, we conclude that, during aging in this animal model, cells other than excitatory neurons provide the primary source of $\mathrm{A} \beta$ peptides that either accrete onto preexisting seeds or serve as templates for additional seeding, aggregation, and subsequent deposition. Additionally, expression of mutant PS1 in these nonexcitatory neuronal cells may also effect conversion of sA $\beta$ monomers to "oligomers" that seed fibril formation and subsequent deposition. Indeed, we documented quantitative differences in the levels of selected cytokines and growth factors secreted by microglia isolated from adult transgenic mice expressing either wild-type human PS1 or mutant PS1 variants (Choi et al., 2008), and it is conceivable that these or other secreted factors may have a critical role in altering $\mathrm{A} \beta$ metabolism in a manner that influences seeding and/or deposition of these pathogenic peptides.

\section{References}

Borchelt DR, Ratovitski T, van Lare J, Lee MK, Gonzales V, Jenkins NA, Copeland NG, Price DL, Sisodia SS (1997) Accelerated amyloid deposition in the brains of transgenic mice coexpressing mutant presenilin 1 and amyloid precursor proteins. Neuron 19:939-945. CrossRef Medline

Choi SH, Veeraraghavalu K, Lazarov O, Marler S, Ransohoff RM, Ramirez JM, Sisodia SS (2008) Non-cell-autonomous effects of presenilin 1 variants on enrichment-mediated hippocampal progenitor cell proliferation and differentiation. Neuron 59:568 -580. CrossRef Medline

Cirrito JR, Yamada KA, Finn MB, Sloviter RS, Bales KR, May PC, Schoepp DD, Paul SM, Mennerick S, Holtzman DM (2005) Synaptic activity regulates interstitial fluid amyloid-beta levels in vivo. Neuron 48:913-922. CrossRef Medline

De Strooper B (2003) Aph-1, Pen-2, and Nicastrin with Presenilin generate an active gamma-secretase complex. Neuron 38:9-12. CrossRef Medline

Forloni G, Demicheli F, Giorgi S, Bendotti C, Angeretti N (1992) Expression of amyloid precursor protein mRNAs in endothelial, neuronal and glial cells: modulation by interleukin-1. Brain Res Mol Brain Res 16:128-134. CrossRef Medline

Golde TE, Estus S, Usiak M, Younkin LH, Younkin SG (1990) Expression of beta amyloid protein precursor mRNAs: recognition of a novel alterna- tively spliced form and quantitation in Alzheimer's disease using PCR. Neuron 4:253-267. CrossRef Medline

Haass C, Hung AY, Selkoe DJ (1991) Processing of beta-amyloid precursor protein in microglia and astrocytes favors an internal localization over constitutive secretion. J Neurosci 11:3783-3793. Medline

Kamenetz F, Tomita T, Hsieh H, Seabrook G, Borchelt D, Iwatsubo T, Sisodia S, Malinow R (2003) APP processing and synaptic function. Neuron 37:925-937. CrossRef Medline

Lah JJ, Heilman CJ, Nash NR, Rees HD, Yi H, Counts SE, Levey AI (1997) Light and electron microscopic localization of presenilin-1 in primate brain. J Neurosci 17:1971-1980. Medline

Mönning U, Sandbrink R, Weidemann A, Banati RB, Masters CL, Beyreuther K (1995) Extracellular matrix influences the biogenesis of amyloid precursor protein in microglial cells. J Biol Chem 270:7104-7110. CrossRef Medline

Price DL, Sisodia SS (1998) Mutant genes in familial Alzheimer's disease and transgenic models. Annu Rev Neurosci 21:479-505. CrossRef Medline

Sherrington R, Rogaev EI, Liang Y, Rogaeva EA, Levesque G, Ikeda M, Chi H, Lin C, Li G, Holman K, Tsuda T, Mar L, Foncin JF, Bruni AC, Montesi MP, Sorbi S, Rainero I, Pinessi L, Nee L, Chumakov I, et al. (1995) Cloning of a gene bearing missense mutations in early-onset familial Alzheimer's disease. Nature 375:754-760. CrossRef Medline

Thinakaran G, Harris CL, Ratovitski T, Davenport F, Slunt HH, Price DL, Borchelt DR, Sisodia SS (1997) Evidence that levels of presenilins (PS1 and PS2) are coordinately regulated by competition for limiting cellular factors. J Biol Chem 272:28415-28422. CrossRef Medline

Tsien JZ, Chen DF, Gerber D, Tom C, Mercer EH, Anderson DJ, Mayford M, Kandel ER, Tonegawa S (1996) Subregion- and cell type-restricted gene knockout in mouse brain. Cell 87:1317-1326. CrossRef Medline

Veeraraghavalu K, Sisodia SS (2013) Mutant presenilin 1 expression in excitatory neurons impairs enrichment-mediated phenotypes of adult hippocampal progenitor cells. Proc Natl Acad Sci U S A 110:9148-9153. CrossRef Medline

Veeraraghavalu K, Zhang C, Miller S, Hefendehl JK, Rajapaksha TW, Ulrich J, Jucker M, Holtzman DM, Tanzi RE, Vassar R, Sisodia SS (2013) Comment on "ApoE-directed therapeutics rapidly clear beta-amyloid and reverse deficits in AD mouse models." Science 340:924-f. CrossRef 